\title{
Performance of the ATLAS Transition Radiation Tracker with Cosmic Rays and First High Energy Collisions at the LHC
}

\author{
James D. Degenhardt for the TRT ATLAS Collaboration
}

\begin{abstract}
The ATLAS Transition Radiation Tracker (TRT) is the outermost of the three sub-systems of the ATLAS Inner Detector at the Large Hadron Collider (LHC) at CERN. It consists of close to 300000 thin-walled drift tubes (straws) providing on average 30 two-dimensional space points with $130 \mu \mathrm{m}$ resolution for charged particle tracks with $|\eta|<2$ and $\mathrm{p}_{\mathrm{T}}>0.5 \mathrm{GeV}$. Along with continuous tracking, it provides particle identification through the detection of transition radiation $x$-ray photons generated by high velocity particles in the many polymer fibers or films that fill the spaces between the straws. The custom-designed radiation-hard front-end electronics implements two thresholds to discriminate the signals: a low threshold $(\sim 300 \mathrm{eV})$ for registering the passage of charged particles, and a high threshold $(\sim 6 \mathrm{keV})$ to flag the absorption of transition radiation $x$-rays. The TRT was successfully commissioned with data collected from several million cosmic ray muons. A special Fast-OR signal from the front-end ASICs was utilized to build a level 1 trigger for cosmic rays traversing the ATLAS Inner Detector which allowed other ATLAS sub-detectors and level 1 triggers to align their timing. The very good timing properties of the TRT detector helped discriminate proton-proton collisions from beam background. This proceeding will describe the operational experiences gained with the ATLAS TRT detector during the commissioning with cosmic rays, and will highlight the excellent performance for charged particle tracking and electron identification based on transition radiation obtained from the first proton-proton collision data at $900 \mathrm{GeV}$ and $7 \mathrm{TeV}$ center-of-mass energy.
\end{abstract}

\section{INTRODUCTION}

A TLAS is a general purpose detector at the Large Hadron Collider (LHC) at CERN. The LHC is a $27 \mathrm{~km}$ circumference, $40 \mathrm{MHz}$ (allowing the design bunch spacing between proton bunches in the accelerator to be $25 \mathrm{~ns}$ ) synchronous proton-proton, or $\mathrm{Pb}-\mathrm{Pb}$ collider. In September 2008, protons were first injected into the LHC at $450 \mathrm{GeV}$, providing ATLAS with the very first single proton beams and beam splashes. Beam splashes are a spray of secondary particles which are produced when one LHC beam, in a controlled manner, is dumped into a closed collimator approximately $140 \mathrm{~m}$ before the beam reaches the ATLAS detector. This creates a synchronous shower of particles that cover the entire cross-section of the ATLAS detector. In October 2008, the LHC was shutdown for repairs for a duration of 14 months and ATLAS began extensive cosmic ray studies. In November 2009, protons were injected into the LHC again, providing

Manuscript received November 21, 2010.

James D. Degenhardt is a post-doctoral fellow with the Physics and Astronomy Department, University of Pennsylvania, Philadelphia, PA 19104 USA, (e-mail: james.degenhardt@cern.ch). another round of single proton beam and beam splashes. On November 23, 2009, proton collisions were first seen at the LHC with a center-of-mass energy of $900 \mathrm{GeV}$. On March 30, 2010, proton collisions at $\sqrt{s}=7 \mathrm{TeV}$ were first observed. Since these first high energy collisions, the LHC has reached a peak instantaneous luminosity of $2 \times 10^{32} \mathrm{~cm}^{-2} \mathrm{~s}^{-1}$ (doubling the goal for the LHC during the 2010 proton physics run). The design instantaneous luminosity for the LHC is $1 \times 10^{34} \mathrm{~cm}^{-2} \mathrm{~s}^{-1}$, which is expected to be reached by the end of the 2011 proton physics run. The LHC has delivered a total of $48 \mathrm{pb}^{-1}$, and ATLAS has recorded $44 \mathrm{pb}^{-1}$ in this $20107 \mathrm{TeV}$ proton physics run.

The ATLAS Transition Radiation Tracker (TRT) is the outermost of the three sub-systems of the ATLAS Inner Detector. The Inner Detector consists of three major sub-systems. The innermost detectors are silicon detectors that provide high resolution spatial measurements of charged particles. The pixel detector, having the highest granularity, surrounds the vertex region of the ATLAS detector. The pixel layer that is closest to the vertex region, called the b-layer, is affixed to the vacuum beam pipe. The silicon strip tracker (SCT), is constructed of stereo layers of fine pitch silicon strip modules and is located between the pixel system and the TRT. As a continuous tracker the TRT provides a large number $(\sim 30)$ of measurement points ("straw hits") on track, with a hit resolution of $130 \mu \mathrm{m}$ for charged particles with $p_{T}>0.5 \mathrm{GeV}$. In addition to significantly contributing to the precision of the momentum measurement, the TRT also provides particle identification information in the form of transition radiation detection and $d E / d x$ measurements for charged particles.

The final piece of the TRT was installed in August, 2008, however the commissioning of the TRT had already begun during the first assembly stages. These early stages of commissioning consisted mainly of checking-out electronics, threshold tuning and determining the time delays. However cosmics were already being observed in the TRT in 2005. During the first injection of protons into the LHC in 2008 and 2009, beam splashes were used to check the timing alignment of the TRT. During the extended cosmic ray running period, the TRT became integral when the TRT Fast-OR cosmic-ray trigger was implemented. Millions of cosmic rays were collected with the trigger, which were essential in aligning and timing-in the ATLAS detector. Then during the LHC commissioning in the autumn of 2009, the good timing properties of the TRT detector was used to confirm the first $p-p$ collisions in the ATLAS detector. Finally, the initial collision runs were used 


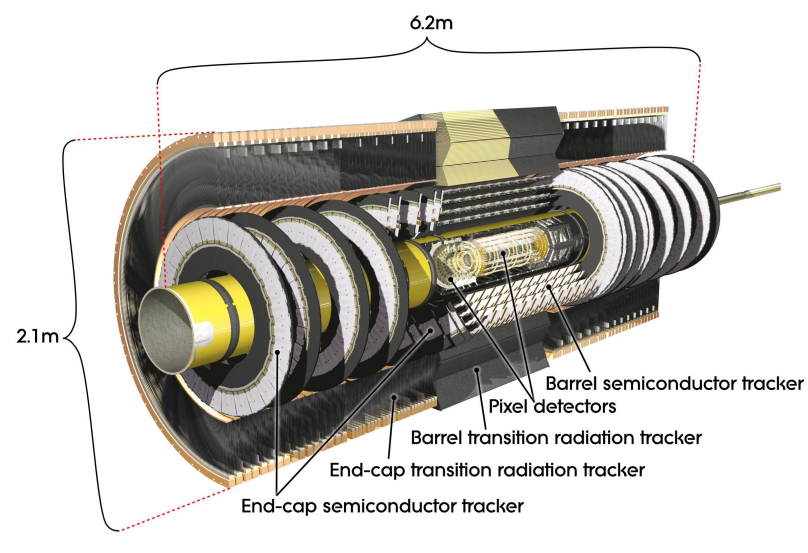

Fig. 1. Cut-away image of the ATLAS Inner Detector

to continue to improve the alignment, as well as calibrate the the response of the TRT to transition radiation photons from electrons and charged hadrons.

This proceeding will first describe the TRT detector in Section II followed by the TRT readout electronics in Section III. Then, in Section IV, a description of the TRT Fast-OR trigger is presented. In Section V, tracking in the TRT is described and subsequently the TRT particle identification in Section VI. Finally the performance of the TRT is reported in Section VII.

\section{Apparatus}

The ATLAS detector [1] at the LHC comprises a thin superconducting solenoid surrounding the Inner Detector and three large superconducting toroids arranged with an eightfold azimuthal coil symmetry placed around the calorimeters, forming the basis of the muon spectrometer.

The layout of the ATLAS Inner Detector is shown in Fig. 1. The Inner-Detector (ID) system is immersed in a $2 \mathrm{~T}$ axial magnetic field and provides tracking information for charged particles in a pseudorapidity range matched by the precision measurements of the electromagnetic calorimeter; the silicon tracking detectors cover the pseudorapidity range $|\eta|<2.5^{1}$. The Transition Radiation Tracker (TRT), which surrounds the silicon detectors, enables track-following up to $|\eta|=2.0$. The entire azimuthal range $(\phi)$ is covered by the entire InnerDetector.

The TRT sensitive volume covers radial distances from $563 \mathrm{~mm}$ to $1066 \mathrm{~mm}$ and is separated into three independent partitions, a barrel and two end-caps. The detector consists of 298304 proportional drift tubes (straws), read out by 350848 channels of electronics. The barrel covers $|\eta|<1$ and is made of 52544 straws $144 \mathrm{~cm}$ in length electrically split in the middle, with independent readout at both ends. The barrel straws are arranged in three cylindrical layers and $32 \phi$ sectors. The transition radiator material which completely surrounds

\footnotetext{
${ }^{1}$ The nominal interaction point is defined as the origin of the coordinate system, while the anti-clockwise beam direction defines the $z$-axis and the $x-y$ plane is transverse to the beam direction. The positive $x$-axis is defined as pointing from the interaction point to the center of the LHC ring and the positive $y$-axis is defined as pointing upwards. The azimuthal angle $\phi$ is measured around the beam axis and the polar angle $\theta$ is the angle from the beam axis. The pseudorapidity is defined as $\eta=-\ln \tan (\theta / 2)$.
}

the straws inside each module consists of polypropylenepolyethylene fiber matting [2]. The two end-caps each contain 122880 straws $37 \mathrm{~cm}$ in length pointing radially outwards from the beam axis. The end-cap straws are arranged in 80 wheellike modular structures that cover the region $1<|\eta|<2$. The TRT end-cap radiators are disk-shaped stacks of alternating layers of thin plastic foils and sheets of a spacer fabric [3]. At roughly $|\eta|=1.5$, the end-caps are divided into two types of wheels (A wheels and B wheels). The A wheels have a smaller spacing between the straw layers (in $z$ ) than the B wheels, and the B wheels also have more foil layers between the straws than the A wheels.

The carbon fiber reinforced straw drift tubes are made of $70 \mu \mathrm{m}$ thick Kapton-based multilayer material [5]. They have an inner diameter of $4 \mathrm{~mm}$ and contain a $31 \mu \mathrm{m}$ diameter, coaxial gold-plated tungsten anode wire. The small tube radius limits the maximum drift time to $\sim 50 \mathrm{~ns}$. The length of the straws has been chosen such that the counting rate per wire does not exceed $20 \mathrm{MHz}$ at the LHC design luminosity. The straw wall, which lies at a potential of $-1.5 \mathrm{kV}$ relative to the wire, provides a separation between the transition radiation medium and the active gas $\left(70 \% \mathrm{Xe}, 27 \% \mathrm{CO}_{2}, 3 \% \mathrm{O}_{2}\right)$. To minimize the number of radiation lengths, only light materials were used throughout the detector.

\section{READOUT AND ELECTRONICS}

When a charged particle traverses a straw it typically loses a $\sim 2 \mathrm{keV}$ of energy to ionization clusters of electrons and ions in the gas. These charged particles are commonly referred to as minimum ionizing particles (MIPs). The liberated electrons drift towards the anode, where the high electric field leads to secondary ionizations in the gas with an average gain of 25000 . This signal is amplified and shaped with an analog Amplifier Shaper Discriminator Base-Line Restorer (ASDBLR) where it is discriminated on a low-threshold (LT) that is typically $\sim 300 \mathrm{eV}$. The much higher energy of the transition radiation photons $(\sim 5-30 \mathrm{keV}$ compared with the MIP energies deposited in the Xe based gas) is detected by a second, high-threshold (HT), discriminator (typically $\sim 6 \mathrm{keV}$, and is recorded in $25 \mathrm{~ns}$ time bins) in the radiation-hard front-end electronics [4]. The signal is then recorded in a digital pipeline of $3.12 \mathrm{~ns}$ wide time bins in a Digital Time Measurement Read-Out Chip (DTMROC) before it is sent to the backend electronics. The DTMROC can further (a) configure the ASDBLR to inject a test pulse used to estimate the effects of radiation damage, (b) sense the low voltage applied to both ASDBLR and DTMROC, (c) transmit configuration and low voltage data to the Trigger, Timing and Control (TTC) back-end during data taking used for e.g. single event upset recovery, and (d) transmit a trigger signal to the TTC back-end (see Section IV).

For the TRT back-end electronics, clock, trigger, and command signals are sent to the front-end boards by TRT-TTC boards, 9U VME modules housed in the counting room. After each trigger, data is read out via the ReadOut Driver (ROD) boards, also 9U VME modules in the counting room. Signals between the front-end and back-end are repeated at TTC and ROD patch panels located in the ATLAS toroid volume. 


\section{FASt-OR COSMIC TRIGger}

When the LHC incident in September 2008 resulted in an extension of the commissioning period using cosmic rays, the decision was made to finalize the implementation of a TRT cosmic trigger. It allowed the TRT to collect cosmic tracks independently of other subsystems, with rates in both barrel and end-cap that approached $20 \mathrm{~Hz}$, while the best rate other triggers could provide for the TRT volume were $\sim 2 \mathrm{~Hz}$. The trigger signal is generated by first OR-ing together all the discriminated channel outputs (either LT or HT) in a DTMROC, then OR-ing together all DTMROCs on a given front-end board [7].

To ensure a high fraction of hits on track $(\sim 30-50 \%)$ while keeping the noise fraction low, the trigger electronics on the DTMROC was configured to generate a trigger signal from the high threshold discriminator that was lowered to minimum ionizing particle levels. This allowed the TRT to perform normal charged particle tracking while simultaneously providing a cosmic muon trigger. As a minor disadvantage, this configuration makes TR calibration difficult and is not compatible with configuration or low voltage data transmission. More than $90 \%$ of the triggers arrive in one clock cycle (where one clock cycle, $25 \mathrm{~ns}$, is the trigger time granularity corresponding the $40 \mathrm{MHz}$ clock of the LHC), enabling the TRT Fast-OR to become a reference for the timing-in of other ATLAS triggers. The trigger helped to improve the barrel muon system trigger timing as well as Inner Detector readout timing, and provided a source of quality tracks for Inner Detector alignment. The barrel trigger rate is $10 \mathrm{~Hz}$ and the efficiency of collecting cosmic tracks is $\sim 75 \%$. The granularity of the trigger is too coarse to make it useful in busier collision events.

\section{TRACKING}

The recorded signals from the wires provide information about both the particle tracks and the synchronization in time of the various readout channels. To discriminate between noise and signals from particles, the energy deposited in the straw is required to be higher than the LT discriminator level. This threshold corresponds to approximately $2 \%$ noise occupancy, and the thresholds are adjusted to create uniform $2 \%$ noise occupancy across all of the channels. The readout timing can be tuned in groups at the level of front-end boards $(\sim 200$ straws) such that all readout signals fit well within the $75 \mathrm{~ns}$ readout window. Figure 2 shows the relative readout timing $\left(T_{0}\right)$ distribution of all barrel and end-cap boards separately, using data from fall 2009 beam splashes. The timing spread was already within \pm 1 ns in the barrel and \pm 3 ns in the endcaps, and the outliers in these histograms have been adjusted as of this writing. Offline, timing variations are corrected on a finer granularity to further improve track reconstruction.

The TRT measures the leading edge (LE) of the signal and the trailing edge (TE) of the signal. The LE of the signal comes from avalanche electrons closest to the wire and is correlated to the distance that these electrons drift from the track to the wire, and hence to the track-to-wire distance. The TE of the signal comes from avalanche electrons furthest away from the wire, or closest to the straw wall, and does

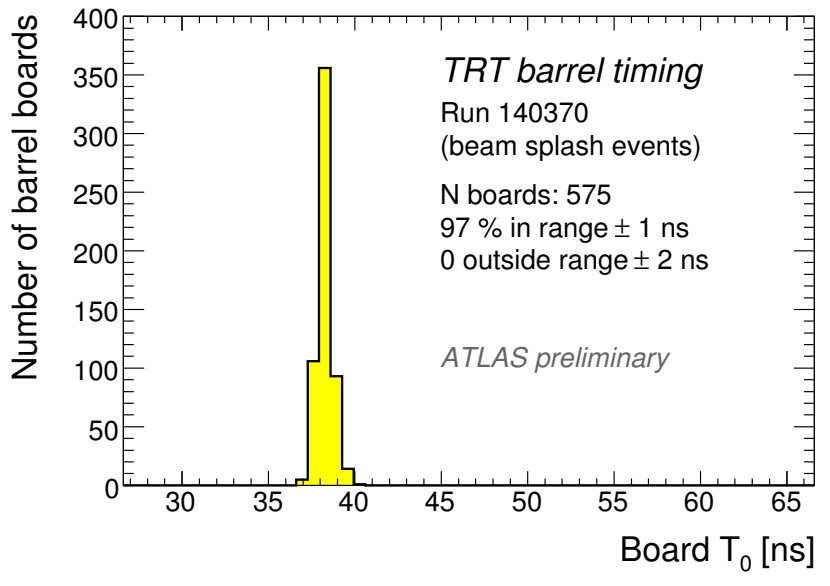

(a) TRT Barrel Board $T_{0}$ from 2009 Beam Splash

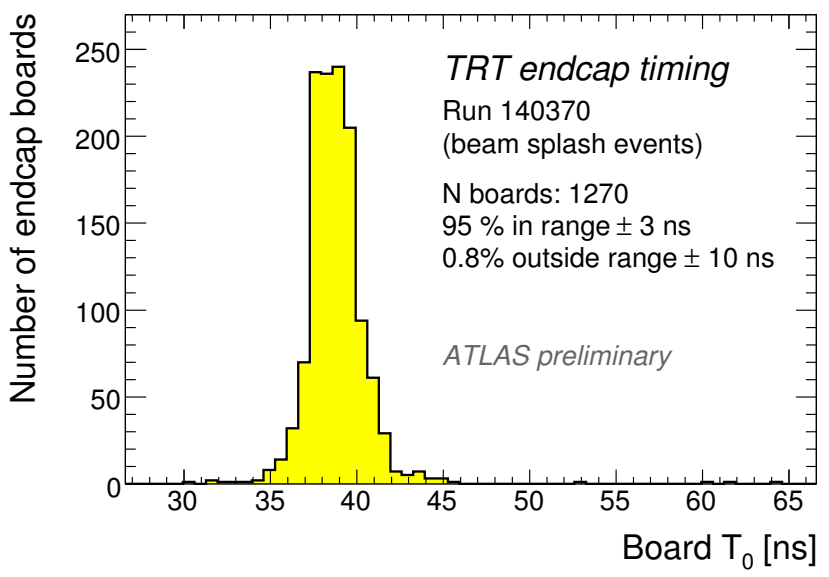

(b) TRT End-cap Board $T_{0}$ from 2009 Beam Splash

Fig. 2. Shown is the distributions of the leading edge time from a single beam splash event from 2009. The timing was measured for groups of straws that share the same TTC line (hardware read-out timing can be adjusted on a TTC line level). One can see that the timing for all boards was already adjusted with very good precision before 2009 beam splash data (following studies using cosmic and 2008 beam splash data). The remaining few outliers were adjusted before the start of collision data taking.

not depend on the track to-wire-distance. So the TE time from multiple hits from the same track arrive at the same time (when taking into account the time of flight (ToF) of the particle). The time over threshold (ToT) is defined as the trailing edge time subtracted from the leading edge time, and can be used as an estimator to measure the amount of energy deposited in the straw. To determine the track position with respect to the wire position, the time of the electrons to drift from the ionization clusters to the wire is determined using the LE. This so-called R-T relation, determined from polynomial fits to data, is used to convert measured drift time into a distance for the track reconstruction algorithms. The detector $T_{0}$, and R-T calibration constants are determined regularly during a 36 hour calibration loop to account for small changes or drifts in 
the constants so that the track reconstruction is not affected by drifts in timing, and gas composition.

With the TRT timing well aligned, the TRT was able to use time of flight (ToF) measurements to confirm the first $p-p$ collisions in the ATLAS detector from November 2009. The TRT read-out timing was first adjusted for collisions (as opposed to cosmic-rays), therefore the hit time measurement was used to check the timing of the collision candidates and single beam events. By using TRT hits that have a ToT $>10 \mathrm{ns,}$ most noise hits are suppressed, and then track reconstruction is not required to make a simple ToF measurement. By plotting the hit position (in the $z$ coordinate) versus the the trailing edge time of the hits, it can be determined if an event was actually a collision (charged particles originating from the vertex region), or a single beam event (charged particles originating upstream of the detector). This behavior is seen in Fig. 3. Beam 1 travels from the positive $z$ direction to the negative $z$ direction. Figure 3(a) shows hits from Beam 1 that appear earlier than they would for collision events. The difference in the measured timing (compared to charged particles originating from collisions) can be used to indicate collisions events from single beam events. A single beam event will have TE times arriving twice as fast as a collision event would, $2 \times z / c=((z / \mathrm{mm}) / 150) \mathrm{ns}$, as indicated on the dashed blue line in the figure. On the negative $z$ side, on the other hand, a single beam event will have similar timing to a collision event. A true collision event timing is shown in Fig. 3(b).

\section{PARTICLE IDENTIFICATION}

The TRT is able to discriminate electrons from other charged particles using transition radiation (TR), which consists of soft x-ray photons emitted by charged particles traversing the boundary between materials with different dielectric constants. The photons are produced with an angle of the order $1 / \gamma$ relative to the track, where $\gamma=E / m c^{2}$, and the number of TR photons produced depends on the $\gamma$ of the particle and the number of boundaries crossed by the particle. TR photons have energies in the range of $\sim 5-30 \mathrm{keV}$, and the TRT xenon gas mixture was chosen to maximize the absorption probability for these photons. Figure 4 shows the probability of a TRT HT hit as a function of $\gamma$. The different radiators used in the barrel (Fig. 4(a)) and the end-caps (Fig. 4(b)) give different onset curve shapes. Not shown in the end-cap plot is the different TR onset curves for the A and B wheels, arising from different spacing of radiator layers as well as a different number of foils between straw layers in $\mathrm{A}$ and $\mathrm{B}$ wheels.

To obtain a pure sample of photons $(>97 \%)$ and a sample dominated by charged hadrons, basic sample selections were required based only on tracking information. A pure sample of electrons is obtained from photon conversions. A sample dominated by hadrons is obtained by selecting all tracks that are not part of a photon conversion. In this sample the particles are assumed to have a mass of a charged pion. As expected from the production of TR, the probability of a HT hit increases for particles with a $\gamma$-factor above 1000, which enables the TRT to separate electrons from hadrons over a

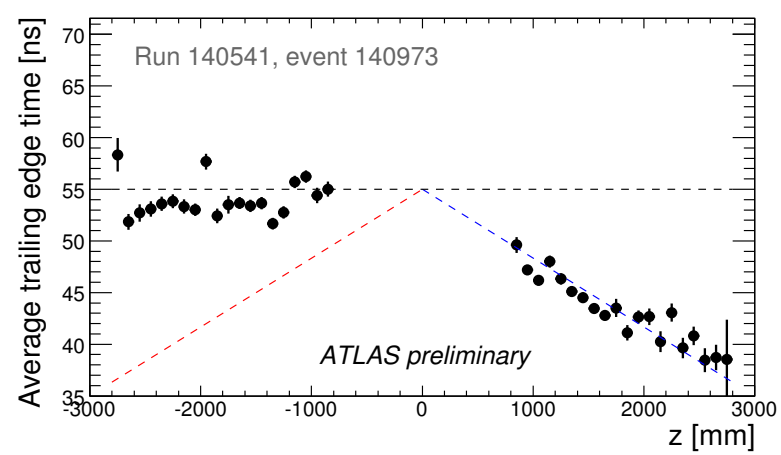

(a) TRT Single Beam Event Time of Flight Measurement

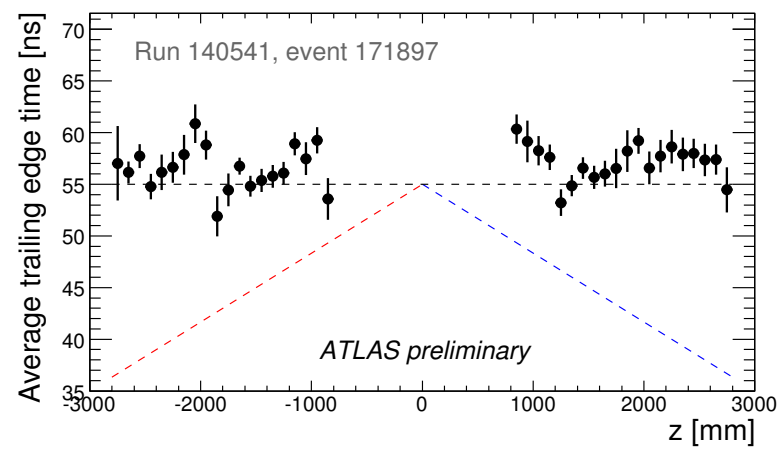

(b) TRT Collision Event Time of Flight Measurement

Fig. 3. Shown in the top figure is a single beam event (beam gas or beam halo) from the LHC Beam 1, which originates from the positive $z$ direction. The hits in the TRT from the trailing edge are early for the positive $z$ side of the TRT, whereas the negative $z$ side hits are in time with a collision event. The bottom plot shows a true collision event where hits on both sides of the TRT detector are in time.

momentum range between $1 \mathrm{GeV}$ and $150 \mathrm{GeV}$, where at low momenta the TRT provides a lager weight in identifying electrons.

The conversion candidates are required to be pairs of oppositely charged tracks, both of which originate from the same vertex, where this vertex is more than $40 \mathrm{~mm}$ away from the beam axis. Both tracks are required to have at least four silicon hits and 20 TRT hits. When one track is identified as an electron (fraction of HT hits $>0.12$ ), the other track is considered to be an electron candidate. The charged hadron candidates are required to have at least one b-layer hit, which greatly suppresses the contamination from conversions and 20 TRT hits.

The number of conversions increase and have a higher momentum spectrum in the end-caps due to the increased amount of material and boost in the forward directions. The results, seen in Fig. 4, indicate that the onset of TR from the lower plateau (corresponding to the probability of producing high-energy delta-rays) and the upper plateau (corresponding to the saturation of the transition radiation production in the geometry chosen for the TRT radiators foils and straw tubes) in the end-cap is steeper than for the barrel. This is expected from 


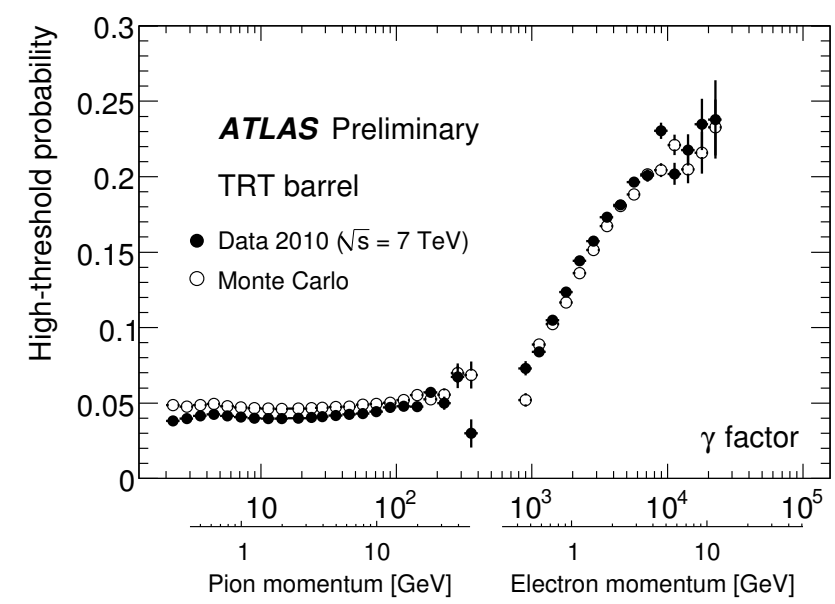

(a) TRT Barrel TR Probability Curve

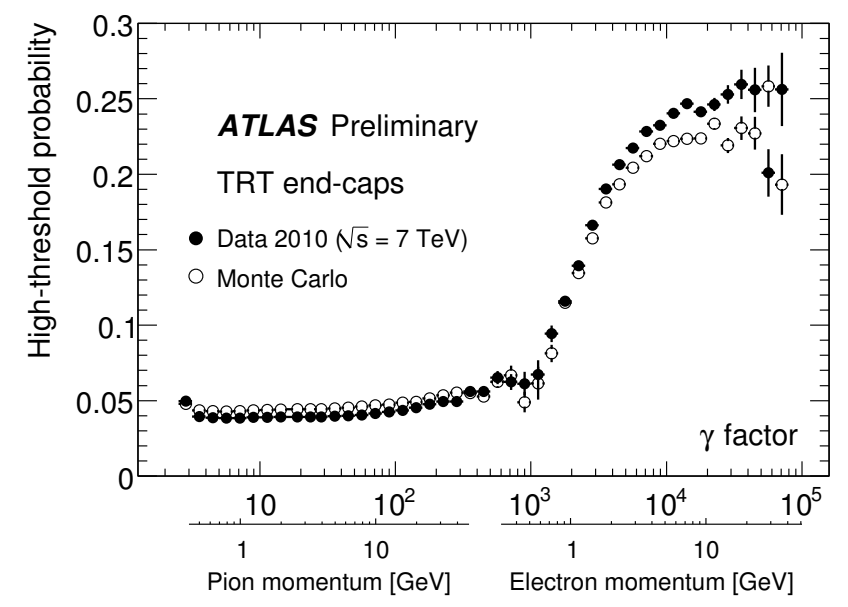

(b) TRT End-cap TR Probability Curve

Fig. 4. Shown are the probability of a TRT high-threshold (HT) hit as a function of the Lorentz factor, $\gamma=E / m$, for the TRT barrel and end-cap regions, as measured in $7 \mathrm{TeV}$ collision events.

the different radiator materials used for the barrel (irregularly spaced fibers) and end-caps (regularly spaced foils).

The results agree with those obtained from minimum bias Monte Carlo simulation for almost all values of gamma and provide the TRT detector with an excellent starting point to study and optimize its particle identification properties. The simulation of the transition radiation has so far been tuned on data collected with barrel modules only in 2004 on a test beam setup and can now be improved based on new results from collision data. The simulated sample is observed to have slightly higher HT probability for hadrons and lower HT probability for electron candidates in the end-caps.

The TRT also uses ToT as an estimator for specific energy loss, $d E / d x$, which can then be used as a particle discriminant. The estimator for specific energy loss is based on the ToT measured by the TRT. The estimator demonstrates the capability to use ToT as a variable for particle identification for heavily ionizing particles. The TRT measures the time when the signal exceeds the LT in bins of $3.12 \mathrm{~ns}$. The estimator is formed using the ToT measurement of all TRT hits on track. As a reference, the average ToT for MIPs (minimum bias tracks between total momenta of $2 \mathrm{GeV}$ and $10 \mathrm{GeV}$ ) is found as a function of the track position in the straw (ToT $\mathrm{T}_{\mathrm{MIP}}$ ). The estimator is then the average sum of the difference between the ToT and this reference, $\sum\left(\mathrm{ToT}-\mathrm{ToT}_{\mathrm{MIP}}\right) / N_{\mathrm{TRT}}$ hits (where $N_{\text {TRT hits }}$ is the number of TRT hits associated to the track). The estimator is offset to be equal to 1 for MIPs. Finally, corrections for variations with pseudo-rapidity are made for hits on tracks in the barrel and end-cap. When plotted versus the total momentum, the $d E / d x$ estimator shows the typical Bethe-Bloch rise for kaons, protons and deuterons. This is seen in Fig. 5, where a sample of approximately 17 million tracks collected during one run of $7 \mathrm{TeV}$ collisions is used. The tracks are required to have at least one pixel, at least six SCT, and at least 15 TRT hits. The range of $0<|\eta|<2$ that is used includes both barrel and end-cap regions of the detector. Track impact parameters are used to select 15.7 million tracks from the primary vertex: $|d 0|<1 \mathrm{~mm}$ and $\left|z_{0} \sin (\theta)\right|<1 \mathrm{~mm}$. Reversing both of these cuts gives 1.7 million tracks not associated with the primary vertex and dominated by secondaries from hadronic interactions and conversions in the beam pipe and detectors. There are far more protons and deuterons than their corresponding antiparticles observed in this sample.

\section{PERFORMANCE}

Many studies have been done using cosmic rays and first collisions data to understand the detector response and maximize the TRT performance. One critical aspect of the TRT performance is, naturally, its availability for physics runs. The TRT has been active and providing good quality data in $100 \%$ of LHC physics runs in 2009 and 2010. In addition to lots of hard work by the TRT operation team, and the implementation of automated procedures to recover from common readout problems contribute to this efficiency.

To provide good momentum resolution, good position resolution is required. Figure 6(a) shows the spatial residual for the barrel and Fig. 6(b) the end-caps. The residuals are unbiased, i.e. the hit measurement from the straw whose residual is calculated is not used in the track fit. The tracks in the sample are from particles at relatively low momentum (a cut $p_{T}>2 \mathrm{GeV}$ was used). Multiple scattering effects are therefore important and lead to residuals larger than the intrinsic resolution. The barrel performance is already close to that expected from Monte Carlo, while the end-caps show a slightly worse resolution. Unlike in the barrel, the rate of tracks in cosmic runs is very small in the end-caps for geometric reasons, hence detailed studies with cosmic ray data were not feasible. More recently, improvements in calibration, tracking and especially alignment have improved these residuals so that the barrel and end-caps are approaching the design resolution of $130 \mu \mathrm{m}$.

The number of hits on a track is important for the momentum measurement, so hit reconstruction efficiency is an 


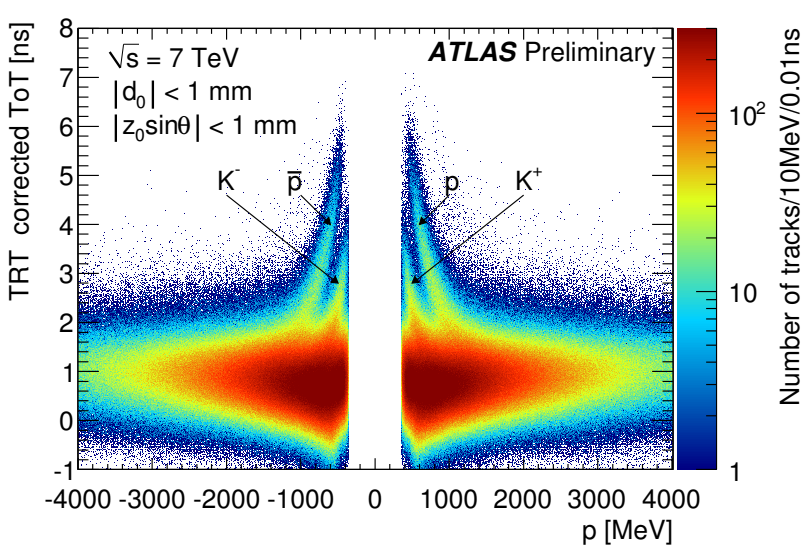

(a) Small Impact Parameter

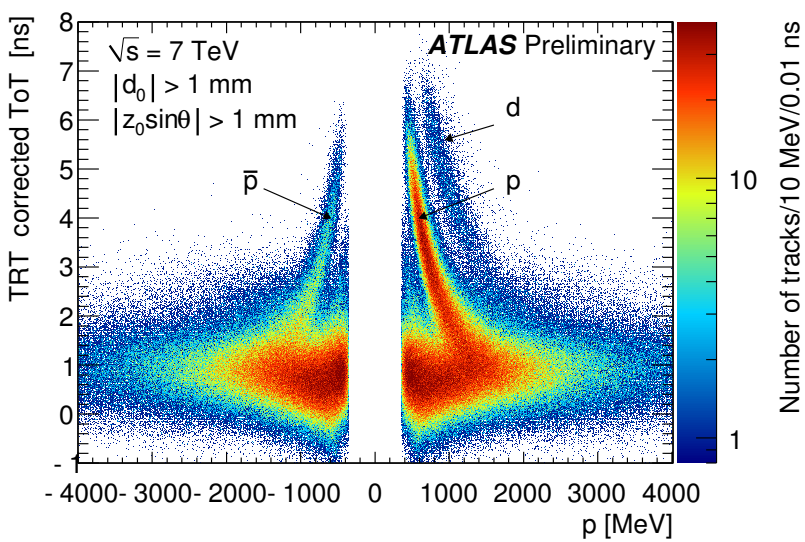

(b) Large Impact Parameter

Fig. 5. Shown are the TRT corrected ToT versus track momentum. One can see the different populations from different particles. For particles with small impact parameter, the kaon and proton distributions are seen. For particles with large impact parameter, one can see the deuterons and protons.

important parameter. The TRT hit efficiency is defined as the number of straws with a hit on track divided by the number of straws crossed by the track, and is shown versus track-towire distance in Fig. 7. The efficiency in the plateau region, or $\pm 1.3 \mathrm{~mm}$ from the straw center, is $94 \%$ and drops off as the track approaches the straw walls where geometric and reconstruction effects start to affect the efficiency measurement. Known dead channels ( $2 \%$ of total) are removed from this plot.

The high hit efficiency and hit residual give a significant contribution on the Inner Detector momentum resolution. From preliminary studies, one can see in Fig. 8 that after including the hit information from the TRT, the the Inner Detector momentum resolution improves, especially at high transverse momenta.

\section{SUMMARY}

This proceeding presents the performance of the ATLAS Transition Radiation Tracker using commissioning and first

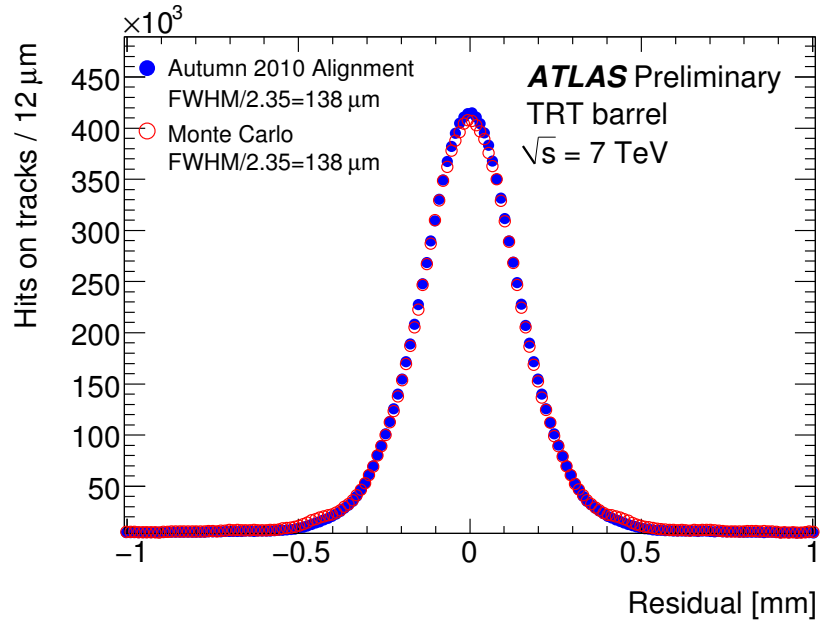

(a) TRT Barrel Unbiased Hit Residuals

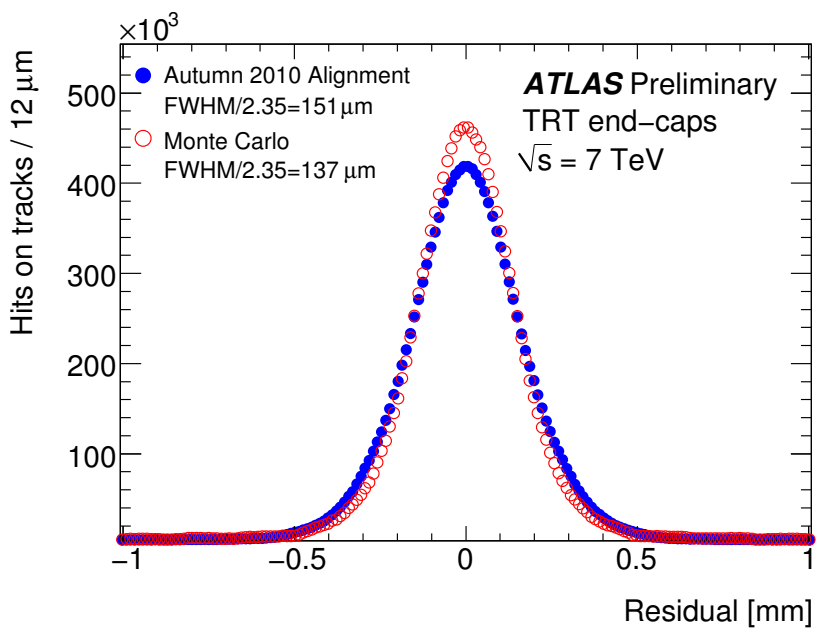

(b) TRT End-cap Unbiased Hit Residuals

Fig. 6. Shown are the TRT unbiased residuals, as obtained from $7 \mathrm{TeV}$ LHC collision data after detector alignment and Monte Carlo (perfectly aligned detector), separately for the barrel and end-caps. The Monte Carlo distributions (open red circles) are normalized to the number of entries in the data (full blue circles). Tracks are required to have $p_{T}>2 \mathrm{GeV}$. For low-momentum tracks, the width of the residual distribution is expected to be larger than the intrinsic accuracy per hit as predicted from the drift time measurement because of the contribution from multiple scattering.

high energy collision data. The TRT has performed well by remaining operational for $100 \%$ of the 2009 and 2010 physics runs, having a better than $94 \%$ hit efficiency, good TR discrimination between charged hadrons and electrons as well as providing particle discrimination based on $d E / d x$. The TRT also provided a pure and efficient cosmic trigger during the cosmic period which became an essential trigger during this period. The latest alignment and calibration studies show that the spatial hit residual of the TRT is approaching the intrinsic resolution of $130 \mu \mathrm{m}$. This leads to a significant improvement of the Inner Detector momentum resolution by the TRT. 


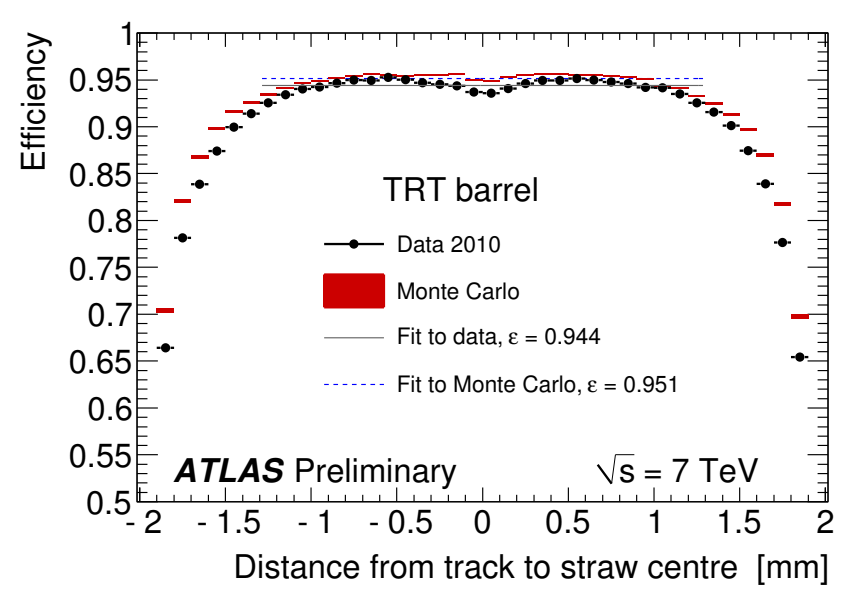

(a) TRT Barrel Hit Efficiency

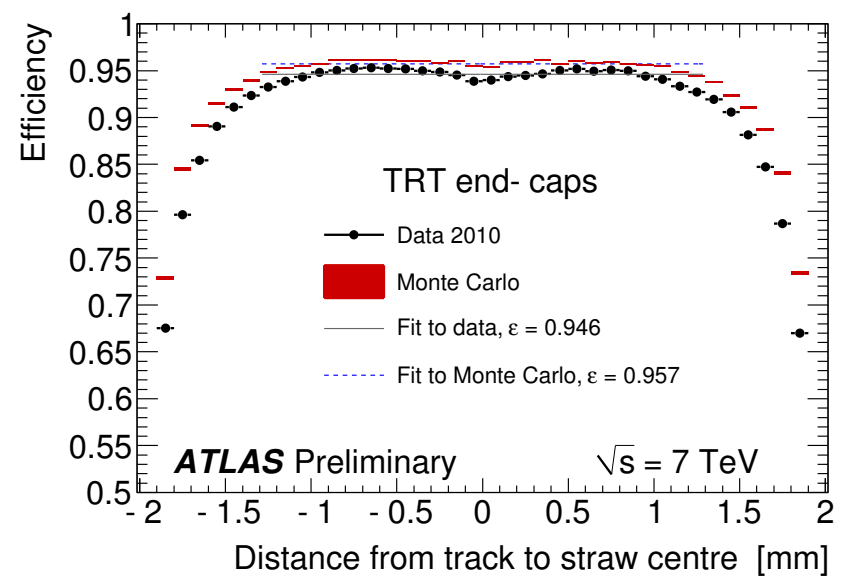

(b) TRT End-cap Hit Efficiency

Fig. 7. Shown is the TRT hit reconstruction efficiency as a function of distance of closest approach of the track to the straw center. The hit reconstruction efficiency is defined as the number of straws with a hit on track divided by the number of straws crossed by the track. Only straws between the first and the last straws with a reconstructed hit on the track are considered in the efficiency calculation, excluding the first and the last. The $2 \%$ of known non-functioning straws are excluded from this study. The tracks are required to have at least one pixel hit, six SCT and 15 TRT hits, as well as $p_{T}>1 \mathrm{GeV},|d 0|<10 \mathrm{~mm}$ and $\left|z_{0}\right|<300 \mathrm{~mm}$. The efficiency for data (MC) is found to be $94 \%(95 \%)$ in the plateau region which is defined by the solid lines. The threshold as simulated in the Monte Carlo is tuned to the data collected during the $900 \mathrm{GeV}$ center of mass collision data.

\section{ACKNOWLEDGMENT}

The ATLAS collaboration wishes to thank CERN for the efficient commissioning and operation of the LHC during this initial high-energy data-taking period as well as the support staff from our institutions without whom ATLAS could not be operated efficiently.

We acknowledge the support of ANPCyT, Argentina; YerPhI, Armenia; ARC, Australia; BMWF, Austria; ANAS, Azerbaijan; SSTC, Belarus; $\mathrm{CNPq}$ and FAPESP, Brazil; NSERC, NRC and CFI, Canada; CERN; CONICYT, Chile;

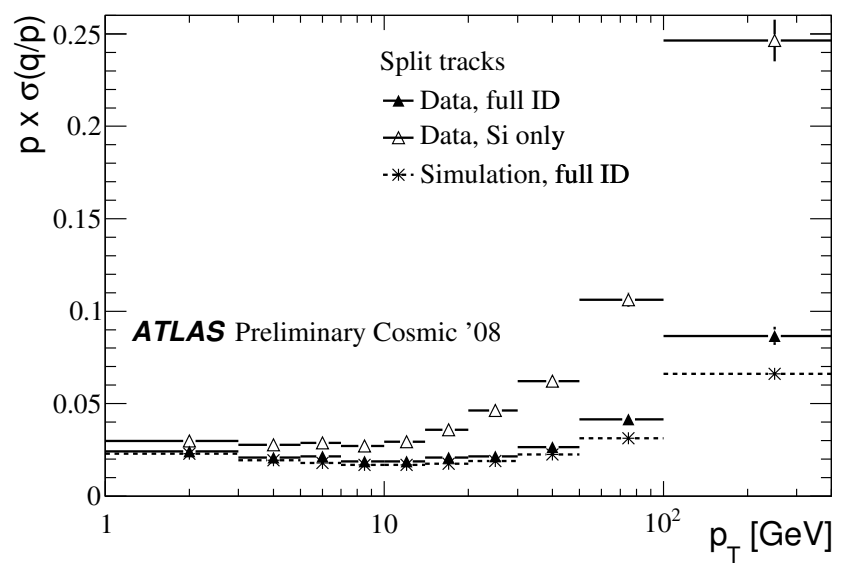

Fig. 8. Relative momentum resolution as a function of $p_{T}$. The relative momentum resolution increases with higher $p_{T}$ due to stiffer tracks and a more difficult measurement of the sagitta. Including information from the TRT extends the lever arm and helps to improve the resolution especially at high $p_{T}$ values. The difference to the MC curve indicates the remaining misalignment.

CAS, MOST and NSFC, China; COLCIENCIAS, Colombia; MEYS (MSMT), MPO and CCRC, Czech Republic; DNRF, DNSRC and Lundbeck Foundation, Denmark; ARTEMIS, European Union; IN2P3-CNRS, CEA-DSM/IRFU, France; GNAS, Georgia; BMBF, DFG, HGF, MPG and AvH Foundation, Germany; GSRT, Greece; ISF, MINERVA, GIF, DIP and Benoziyo Center, Israel; INFN, Italy; MEXT and JSPS, Japan; CNRST, Morocco; FOM and NWO, Netherlands; RCN, Norway; MNiSW, Poland; GRICES and FCT, Portugal; MERYS (MECTS), Romania; MES of Russia and ROSATOM, Russian Federation; JINR; MSTD, Serbia; MSSR, Slovakia; ARRS and MVZT, Slovenia; DST/NRF, South Africa; MICINN, Spain; SRC and Wallenberg Foundation, Sweden; SER, SNSF and Cantons of Bern and Geneva, Switzerland; NSC, Taiwan; TAEK, Turkey; STFC, the Royal Society and Leverhulme Trust, United Kingdom; DOE and NSF, United States of America.

\section{REFERENCES}

[1] The ATLAS Collaboration, The ATLAS Experiment at the CERN Large Hadron Collider, JINST 3 (2008) S08003.

[2] E. Abat et al., The ATLAS TRT Barrel Detector, JINST 3 (2008) P02014.

[3] E. Abat et al., The ATLAS TRT end-cap detectors, JINST 3 (2008) P10003.

[4] E. Abat et al., The ATLAS TRT electronics, JINST 3 (2008) P06007.

[5] E. Abat et al., The ATLAS Transition Radiation Tracker (TRT) proportional drift-tube: Design and Performance, JINST 3 (2008) P02013.

[6] The ATLAS Collaboration, The ATLAS Inner Detector commissioning and calibration (2009) (ONLINE) http: //arxiv.org/abs/1004.5293.

[7] S. Fratina et al., The ATLAS Transition Radiation Detector (TRT) Fast-OR Trigger (2009) (ONLINE) http: //cdsweb.cern.ch/record/1229213/files /ATL-INDET-PUB-2009-002.pdf. 